\title{
Thermodynamics of Hydronium, Zundel and Eigen ions
}

\author{
Nikolai Mikhailovich Bazhin ${ }^{1 *}$, Kelath Murali Manoj ${ }^{2}$ \\ (*Corresponding author)
}

\author{
${ }^{1}$ Institute of Chemical Kinetics and Combustion \\ Russian Academy of Sciences, St. Institutskaya 3, 630090 Novosibirsk, Russia. \\ Email: bazhin8999@kinetics.nsc.ru (ORCID: 0000-0002-1048-303X)
}

${ }^{2}$ Satyamjayatu: The Science \& Ethics Foundation,

Shoranur-2 (PO), Palakkad District, Kerala, India-679122.

Email: murman@satyamjayatu.com (ORCID: 0000-0003-4515-994X)

\begin{abstract}
The heterolytic dissociation of water gives the cation of proton, which has fleeting existence in reality. It exists in aqueous solution in various levels of hydration, as hydronium $\left(\mathrm{H}_{3} \mathrm{O}^{+}\right)$, Zundel $\left(\mathrm{H}_{5} \mathrm{O}_{2}{ }^{+}\right)$and Eigen $\left(\mathrm{H}_{9} \mathrm{O}_{4}{ }^{+}\right)$ions. Herein, we present the thermodynamic parameters involved in the overall treatment. These values are crucial in understanding the behaviour of water and protons in cellular physiology at interfaces and bulk.
\end{abstract}

Keywords: proton; water; hydronium; Zundel ion; Eigen ion; solvation 
The biological solvent of water has a low dissociation, and this is very important towards explaining several physiological activities. Herein, we present the values of thermodynamica constants for the various species, in continuing efforts to understand the dynamics of bioenergetics.

\section{1. $\mathrm{H}_{3} \mathrm{O}^{+}$}

1.1 The thermodynamic parameters for $\mathrm{H}_{2} \mathrm{O}_{\text {gas }}$ and $\mathrm{H}_{\text {gas }}^{+}$

The used thermodynamic parameters for $\mathrm{H}_{2} \mathrm{O}_{\text {gas }}$ and $\mathrm{H}_{\text {gas }}^{+}$are taken from $[1,2]$
$\mathrm{A}_{\mathrm{st}} \rightarrow \mathrm{H}_{2} \mathrm{O}_{\text {gas }}$
$\Delta_{\mathrm{f}} H^{\mathrm{o}}=-241.8$
$\Delta_{\mathrm{f}} G^{\mathrm{o}}=-228.6 \mathrm{~kJ} / \mathrm{mol}$
$\mathrm{A}_{\mathrm{st}} \rightarrow \mathrm{H}_{\mathrm{gas}}^{+}+\mathrm{e}_{\mathrm{gas}}^{-}$
$\Delta_{\mathrm{f}} H^{\circ}=1536.2$
$\Delta_{\mathrm{f}} G^{\mathrm{o}}=1517 \mathrm{~kJ} / \mathrm{mol}$

Parameters $\mathrm{H}_{3} \mathrm{O}^{+}$formation reaction in the gas phase are taken from [1, Table 10-168]:

$$
\mathrm{H}_{2} \mathrm{O}_{\text {gas }}+\mathrm{H}_{\text {gas }}^{+} \rightarrow \mathrm{H}_{3} \mathrm{O}_{\text {gas }}^{+} \quad \Delta_{\mathrm{r}} H^{\mathrm{o}}=-690.36 \quad \Delta_{\mathrm{r}} G^{\mathrm{o}}=-660.0 \mathrm{~kJ} / \mathrm{mol}
$$

1.2. The thermodynamic functions of the formation of $\mathrm{H}_{3} \mathrm{O}_{\text {gas }}^{+}$

Let us now find the values of the thermodynamic functions of the formation of ions $\mathrm{H}_{3} \mathrm{O}_{\text {gas }}^{+}$in the gas phase. From the last equation $\left(\mathrm{H}_{2} \mathrm{O}_{\text {gas }}+\mathrm{H}_{\text {gas }}^{+} \rightarrow \mathrm{H}_{3} \mathrm{O}_{\text {gas }}^{+}\right)$we find

$$
\begin{aligned}
\Delta_{\mathrm{r}} H^{\mathrm{o}}= & \Delta_{\mathrm{f}} H^{\mathrm{o}}\left(\mathrm{H}_{3} \mathrm{O}_{\mathrm{gas}}^{+}\right)-\Delta_{\mathrm{f}} H^{\mathrm{o}}\left(\mathrm{H}_{\mathrm{gas}}^{+}\right)-\Delta_{\mathrm{f}} H^{\mathrm{o}}\left(\mathrm{H}_{2} \mathrm{O}_{\mathrm{gas}}\right)= \\
& -690.36=\Delta_{\mathrm{f}} H^{\mathrm{o}}\left(\mathrm{H}_{3} \mathrm{O}_{\text {gas }}^{+}\right)-1536.2+241.8
\end{aligned}
$$

and

$$
\Delta_{\mathrm{f}} H^{\mathrm{o}}\left(\mathrm{H}_{3} \mathrm{O}_{\mathrm{gas}}^{+}\right)=-690.36+1536.2-241.8=604.04 \mathrm{~kJ} / \mathrm{mol}
$$

Similarly, we find the values of the Gibbs function

$$
\begin{aligned}
\Delta_{\mathrm{r}} G^{\mathrm{o}}= & \Delta_{\mathrm{f}} G^{\mathrm{o}}\left(\mathrm{H}_{3} \mathrm{O}_{\mathrm{gas}}^{+}\right)-\Delta_{\mathrm{f}} G^{\mathrm{o}}\left(\mathrm{H}_{\mathrm{gas}}^{+}\right)-\Delta_{\mathrm{f}} G^{\mathrm{o}}\left(\mathrm{H}_{2} \mathrm{O}_{\mathrm{gas}}\right)= \\
& -660=\Delta_{\mathrm{f}} G^{\mathrm{o}}\left(\mathrm{H}_{3} \mathrm{O}_{\text {gas }}^{+}\right)-1517+228.6 \text { and } \\
& \Delta_{\mathrm{f}} G^{\mathrm{o}}\left(\mathrm{H}_{3} \mathrm{O}_{\text {gas }}^{+}\right)=-660+1517-228.6=628.4 \mathrm{~kJ} / \mathrm{mol}
\end{aligned}
$$

\subsection{Solvation}

We will consider the process of solvation of a proton in the form of two reactions: the addition of a proton to a water molecule in the gas phase and the solvation of the formed ion $\mathrm{H}_{3} \mathrm{O}_{\text {gas }}^{+}$.

In the case of enthalpy we have

$$
\Delta_{\mathrm{S}} H^{\mathrm{o}}\left(\mathrm{H}^{+}\right)=\Delta_{\mathrm{r}} H^{\mathrm{o}}\left(\mathrm{H}_{2} \mathrm{O}+\mathrm{H}^{+}\right)_{\text {gas }}+\Delta_{\mathrm{S}} H^{\mathrm{o}}\left(\mathrm{H}_{3} \mathrm{O}^{+}\right)=-690.36+\Delta_{\mathrm{S}} H^{\mathrm{o}}\left(\mathrm{H}_{3} \mathrm{O}^{+}\right)
$$


From the last equation we obtain

$$
\Delta_{\mathrm{S}} H^{\mathrm{o}}\left(\mathrm{H}_{3} \mathrm{O}^{+}\right)=\Delta_{\mathrm{S}} H^{\mathrm{o}}\left(\mathrm{H}^{+}\right)+690.36=-1150.1+690.36=-459.74 \mathrm{~kJ} / \mathrm{mol}
$$

Carrying out similar calculations for the Gibbs function,

$\Delta_{\mathrm{S}} G^{\mathrm{o}}\left(\mathrm{H}^{+}\right)=-1104.5=\Delta_{\mathrm{S}} G^{\mathrm{o}}\left(\mathrm{H}_{3} \mathrm{O}^{+}\right)+\Delta_{\mathrm{r}} G^{\mathrm{o}}\left(\mathrm{H}_{2} \mathrm{O}+\mathrm{H}^{+}\right)=\Delta_{\mathrm{S}} G^{\mathrm{o}}\left(\mathrm{H}_{3} \mathrm{O}^{+}\right)-660$.

we find

$$
\Delta_{\mathrm{S}} G^{\mathrm{o}}\left(\mathrm{H}_{3} \mathrm{O}^{+}\right)=\Delta_{\mathrm{S}} G^{\mathrm{o}}\left(\mathrm{H}^{+}\right)+660=-1104.5+660=-444.5 \mathrm{~kJ} / \mathrm{mol} .
$$

1.4. The thermodynamic parameters of $\mathrm{H}_{3} \mathrm{O}^{+}$aq

Knowing the thermodynamic parameters of solvation and thermodynamic parameters in the gas phase we calculate the standard values of the thermodynamic parameters formation for $\mathrm{H}_{3} \mathrm{O}_{\text {aq }}^{+}$in an aqueous solution.

$\Delta_{\mathrm{S}} H^{\mathrm{o}}\left(\mathrm{H}_{3} \mathrm{O}^{+}\right)=\Delta_{\mathrm{f}} H^{\mathrm{o}}\left(\mathrm{H}_{3} \mathrm{O}^{+}{ }_{\mathrm{aq}}\right)-\Delta_{\mathrm{f}} H^{\mathrm{o}}\left(\mathrm{H}_{3} \mathrm{O}_{\text {gas }}^{+}\right)$and

$\Delta_{\mathrm{f}} H^{\mathrm{o}}\left(\mathrm{H}_{3} \mathrm{O}^{+}{ }_{\mathrm{aq}}\right)=\Delta_{\mathrm{S}} H^{\mathrm{o}}\left(\mathrm{H}_{3} \mathrm{O}^{+}\right)+\Delta_{\mathrm{f}} H^{\mathrm{o}}\left(\mathrm{H}_{3} \mathrm{O}_{\text {gas }}^{+}\right)=-459.74+604.04=144.3 \mathrm{~kJ} / \mathrm{mol}$

For $G$ function we have

$\Delta_{\mathrm{S}} G^{\mathrm{o}}\left(\mathrm{H}_{3} \mathrm{O}^{+}\right)=\Delta_{\mathrm{f}} G^{\mathrm{o}}\left(\mathrm{H}_{3} \mathrm{O}^{+}{ }_{\mathrm{aq}}\right)-\Delta_{\mathrm{f}} G^{\mathrm{o}}\left(\mathrm{H}_{3} \mathrm{O}_{\text {gas }}^{+}\right)$and

$\Delta_{\mathrm{f}} G^{\mathrm{o}}\left(\mathrm{H}_{3} \mathrm{O}^{+}{ }_{\mathrm{aq}}\right)=\Delta_{\mathrm{S}} G^{\mathrm{o}}\left(\mathrm{H}_{3} \mathrm{O}^{+}\right)+\Delta_{\mathrm{f}} G^{\mathrm{o}}\left(\mathrm{H}_{3} \mathrm{O}_{\text {gas }}^{+}\right)=-444.5+628.4=183.9 \mathrm{~kJ} / \mathrm{mol}$.

1.5. Transformed value

Transformed value is equal

$\Delta_{\mathrm{f}} G^{\mathrm{oo}}\left(\mathrm{H}_{3} \mathrm{O}^{+}{ }_{\mathrm{aq}}\right)=\Delta_{\mathrm{f}} G^{\mathrm{o}}\left(\mathrm{H}_{3} \mathrm{O}^{+}{ }_{\mathrm{aq}}\right)+121.49[3]=183.9+121.49=305.39 \mathrm{~kJ} / \mathrm{mol}$

1.6. Convention values

Knowing the standard thermodynamic parameters of $\mathrm{H}_{3} \mathrm{O}^{+}$aq formation, we find similar values within the framework of the convention [2]

$\Delta_{\mathrm{f}} H_{\text {conv }}^{\mathrm{O}}\left(\mathrm{H}_{3} \mathrm{O}^{+}{ }_{\mathrm{aq}}\right)=\Delta_{\mathrm{f}} H^{\mathrm{o}}\left(\mathrm{H}_{3} \mathrm{O}^{+}{ }_{\mathrm{aq}}\right)-386.25=144.3-386.25=-241.95 \mathrm{~kJ} / \mathrm{mol}$ and $\Delta_{\mathrm{f}} G_{\text {conv }}^{\mathrm{o}}\left(\mathrm{H}_{3} \mathrm{O}^{+}{ }_{\mathrm{aq}}\right)=\Delta_{\mathrm{f}} G^{\mathrm{o}}\left(\mathrm{H}_{3} \mathrm{O}^{+}{ }_{\mathrm{aq}}\right)-412.5=183.9-412.5=-228.6 \mathrm{~kJ} / \mathrm{mol}$ 


\section{Zundel ion}

2.1. The thermodynamic parameters for $\mathrm{H}_{5} \mathrm{O}_{2 \text { gas }}^{+}$

Zundel ion is $\mathrm{H}_{5} \mathrm{O}_{2}^{+}=\mathrm{H}^{+} \ldots 2 \mathrm{H}_{2} \mathrm{O}$.

We write the formation of an Zundel ion in gas phase in the form of two reactions

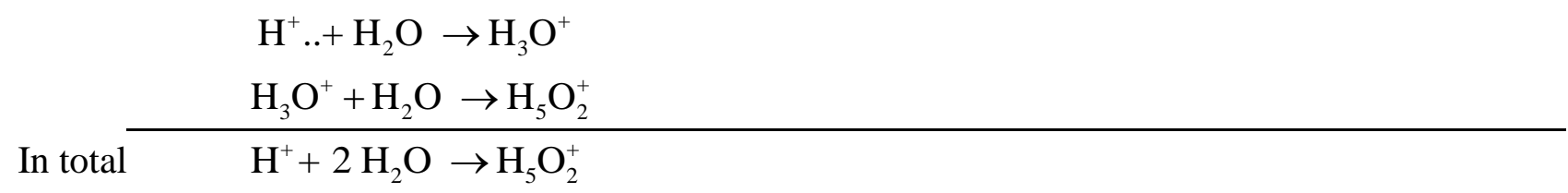

From [4], we take data from Table 1

Table I. Experimental Thermodynamic Values for the Gas-Phase Reactions

$\mathrm{H}_{2} \mathrm{O}+\mathrm{H}^{+}\left(\mathrm{H}_{2} \mathrm{O}\right)_{\mathrm{n}-1} \square \quad \mathrm{H}^{+}\left(\mathrm{H}_{2} \mathrm{O}\right)_{\mathrm{n}}, \quad(\mathrm{n}-1, \mathrm{n})[4]$

\begin{tabular}{|c|c|c|c|c|}
\hline$(\mathrm{n}-1, \mathrm{n})$ & $-\Delta H_{n-1 . n} \mathrm{kcal} / \mathrm{mol}$ & $-\Delta H_{n-1 . n} \mathrm{~kJ} / \mathrm{mol}$ & $-\Delta G_{n-1 . n} \mathrm{kcal} / \mathrm{mol}$ & $-\Delta G_{n-1 . n} \mathrm{~kJ} / \mathrm{mol}$ \\
\hline 0,1 & 165 & 690.36 & & $660[1]$ \\
\hline 1,2 & 36 & 150.62 & 25 & 104.6 \\
\hline 2,3 & 22.3 & 93.3 & 13.6 & 56.9 \\
\hline 3,4 & 17 & 71.13 & 8.5 & 35.56 \\
\hline
\end{tabular}

Hence, $\Delta_{\mathrm{r}} H\left(\mathrm{H}^{+}+2 \mathrm{H}_{2} \mathrm{O} \rightarrow \mathrm{H}_{5} \mathrm{O}_{2}^{+}\right)_{\text {gas }}=-690.36-150.62=-840.98 \mathrm{~kJ} / \mathrm{mol}$ and

$$
\Delta_{\mathrm{r}} G\left(\mathrm{H}^{+}+2 \mathrm{H}_{2} \mathrm{O} \rightarrow \mathrm{H}_{5} \mathrm{O}_{2}^{+}\right)_{\text {gas }}=-660-104.6=-764.6 \mathrm{~kJ} / \mathrm{mol}
$$

2.2. The thermodynamic functions of $\mathrm{H}_{5} \mathrm{O}_{2 \mathrm{gas}}^{+}$

For the reaction of formation of $\mathrm{H}_{5} \mathrm{O}_{2 g a s}^{+},\left(\mathrm{H}^{+}+2 \mathrm{H}_{2} \mathrm{O} \rightarrow \mathrm{H}_{5} \mathrm{O}_{2}^{+}\right)_{\text {gas }}$, we write

$\Delta_{\mathrm{r}} H^{\mathrm{o}}=\Delta_{\mathrm{f}} H^{\circ}\left(\mathrm{H}_{5} \mathrm{O}_{2 \mathrm{gas}}^{+}\right)-\Delta_{\mathrm{f}} H^{\circ}\left(\mathrm{H}_{\mathrm{gas}}^{+}\right)-2 \Delta_{\mathrm{f}} H^{\circ}\left(\mathrm{H}_{2} \mathrm{O}_{\text {gas }}\right)=$

$$
-840.98=\Delta_{\mathrm{f}} H^{\circ}\left(\mathrm{H}_{5} \mathrm{O}_{2 \mathrm{gas}}^{+}\right)-1536.2+2 \cdot 241.8 \text { and }
$$

Hence

$$
\Delta_{\mathrm{f}} H^{\circ}\left(\mathrm{H}_{5} \mathrm{O}_{2 \mathrm{gas}}^{+}\right)=-840.98+1536.2-2241.8=211.62 \mathrm{~kJ} / \mathrm{mol}
$$

Similarly, we find the values of the Gibbs function

$$
\begin{aligned}
\Delta_{\mathrm{r}} G^{\mathrm{o}}= & \Delta_{\mathrm{f}} G^{\mathrm{o}}\left(\mathrm{H}_{5} \mathrm{O}_{2 \mathrm{gas}}^{+}\right)-\Delta_{\mathrm{f}} G^{\mathrm{o}}\left(\mathrm{H}_{\mathrm{gas}}^{+}\right)-2 \cdot \Delta_{\mathrm{f}} G^{\mathrm{o}}\left(\mathrm{H}_{2} \mathrm{O}_{\mathrm{gas}}\right)= \\
& -764.6=\Delta_{\mathrm{f}} G^{\mathrm{o}}\left(\mathrm{H}_{5} \mathrm{O}_{2 \mathrm{gas}}^{+}\right)-1517+2 \cdot 228.6 \text { and }
\end{aligned}
$$




$$
\Delta_{\mathrm{f}} G^{\mathrm{o}}\left(\mathrm{H}_{5} \mathrm{O}_{2 \mathrm{gas}}^{+}\right)=-764.6+1517-2 \cdot 228.6=295.2 \mathrm{~kJ} / \mathrm{mol}
$$

\subsection{Solvation}

We will consider the process of solvation of a proton in the form of two reactions: the addition of a proton to a two water molecules in the gas phase and the solvation of the formed ion $\mathrm{H}_{5} \mathrm{O}_{2 \mathrm{gas}}^{+}$.

In the case of enthalpy we have

$$
\Delta_{\mathrm{S}} H^{\mathrm{o}}\left(\mathrm{H}^{+}\right)=\Delta_{\mathrm{r}} H^{\mathrm{o}}\left(\mathrm{H}^{+}+2 \mathrm{H}_{2} \mathrm{O}\right)_{\mathrm{gas}}+\Delta_{\mathrm{S}} H^{\mathrm{o}}\left(\mathrm{H}_{5} \mathrm{O}_{2}^{+}\right)=-840.98+\Delta_{\mathrm{S}} H^{\mathrm{o}}\left(\mathrm{H}_{5} \mathrm{O}_{2}^{+}\right) .
$$

From the last equation we obtain

$$
\Delta_{\mathrm{S}} H^{\mathrm{o}}\left(\mathrm{H}_{5} \mathrm{O}_{2}^{+}\right)=\Delta_{\mathrm{S}} H^{\mathrm{o}}\left(\mathrm{H}^{+}\right)+840.98=-1150.1+840.98=-309.12 \mathrm{~kJ} / \mathrm{mol}
$$

Carrying out similar calculations for the Gibbs function,

$$
\begin{gathered}
\Delta_{\mathrm{S}} G^{\mathrm{o}}\left(\mathrm{H}^{+}\right)=-1104.5=\Delta_{\mathrm{r}} G^{\mathrm{o}}\left(\mathrm{H}^{+}+2 \mathrm{H}_{2} \mathrm{O} \rightarrow \mathrm{H}_{5} \mathrm{O}_{2}^{+}\right)_{\text {gas }}+\Delta_{\mathrm{S}} G^{\mathrm{o}}\left(\mathrm{H}_{5} \mathrm{O}_{2}^{+}\right)= \\
\Delta_{\mathrm{S}} G^{\mathrm{o}}\left(\mathrm{H}_{5} \mathrm{O}_{2}^{+}\right)-764.6 \mathrm{~kJ} / \mathrm{mol} .
\end{gathered}
$$

we find

$$
\Delta_{\mathrm{S}} G^{\mathrm{o}}\left(\mathrm{H}_{5} \mathrm{O}_{2}^{+}\right)=-1104.5+764.6=-333.9 \mathrm{~kJ} / \mathrm{mol} \text {. }
$$

2.4. The thermodynamic parameters of $\mathrm{H}_{5} \mathrm{O}_{2 \mathrm{aq}}^{+}$

Knowing the thermodynamic parameters of solvation and thermodynamic parameters in the gas phase we calculate the standard values of the thermodynamic parameters formation for $\mathrm{H}_{5} \mathrm{O}_{2}^{+}$aq in an aqueous solution.

$\Delta_{\mathrm{S}} H^{\mathrm{o}}\left(\mathrm{H}_{5} \mathrm{O}_{2}^{+}\right)=\Delta_{\mathrm{f}} H^{\mathrm{o}}\left(\mathrm{H}_{5} \mathrm{O}_{2}^{+}\right.$aq $)-\Delta_{\mathrm{f}} H^{\mathrm{o}}\left(\mathrm{H}_{5} \mathrm{O}_{2 \text { gas }}^{+}\right)$and

$\Delta_{\mathrm{f}} H^{\mathrm{o}}\left(\mathrm{H}_{5} \mathrm{O}_{2}^{+}\right.$aq $)=\Delta_{\mathrm{S}} H^{\mathrm{o}}\left(\mathrm{H}_{5} \mathrm{O}_{2}^{+}\right)+\Delta_{\mathrm{f}} H^{\mathrm{o}}\left(\mathrm{H}_{5} \mathrm{O}_{2}^{+}\right.$gas $)=-309.12+211.62=-97.5 \mathrm{~kJ} / \mathrm{mol}$

For $G$ function we have

$\Delta_{\mathrm{S}} G^{\mathrm{o}}\left(\mathrm{H}_{5} \mathrm{O}_{2}^{+}\right)=\Delta_{\mathrm{f}} G^{\mathrm{o}}\left(\mathrm{H}_{5} \mathrm{O}_{2}^{+}\right.$aq $)-\Delta_{\mathrm{f}} G^{\mathrm{o}}\left(\mathrm{H}_{5} \mathrm{O}_{2}^{+}\right.$gas $)$and

$\Delta_{\mathrm{f}} G^{\mathrm{o}}\left(\mathrm{H}_{5} \mathrm{O}_{2}^{+}\right.$aq $)=\Delta_{\mathrm{S}} G^{\mathrm{o}}\left(\mathrm{H}_{5} \mathrm{O}_{2}^{+}\right.$aq $)+\Delta_{\mathrm{f}} G^{\mathrm{o}}\left(\mathrm{H}_{5} \mathrm{O}_{2}^{+}\right.$gas $)=-333.9+295.2=-38.7 \mathrm{~kJ} / \mathrm{mol}$

\subsection{Transformed value}

Transformed value is equal

$\Delta_{\mathrm{f}} G^{\mathrm{o}}\left(\mathrm{H}_{5} \mathrm{O}_{2}^{+}\right.$aq $)=\Delta_{\mathrm{f}} G^{\mathrm{o}}\left(\mathrm{H}_{5} \mathrm{O}_{2}^{+}\right.$aq $)+121.49[3]=-38.7+203.03=164.33 \mathrm{~kJ} / \mathrm{mol}$ 
2.6. Convention values

Knowing the standard thermodynamic parameters of $\mathrm{H}_{5} \mathrm{O}_{2}^{+}$aq formation, we find similar values within the framework of the convention

$$
\begin{aligned}
& \Delta_{\mathrm{f}} H_{\text {conv }}^{\mathrm{o}}\left(\mathrm{H}_{5} \mathrm{O}_{2 \text { aq }}^{+}\right)=\Delta_{\mathrm{f}} H^{\mathrm{o}}\left(\mathrm{H}_{5} \mathrm{O}_{2}^{+} \text {aq }\right)-386.25=-97.5-386.25=-483.75 \mathrm{~kJ} / \mathrm{mol} \text { and } \\
& \Delta_{\mathrm{f}} G_{\text {conv }}^{\mathrm{o}}\left(\mathrm{H}_{5} \mathrm{O}_{2 \text { aq }}^{+}\right)=\Delta_{\mathrm{f}} G^{\mathrm{o}}\left(\mathrm{H}_{5} \mathrm{O}_{2}^{+} \text {aq }\right)-412.5=-38.7-412.5=-451.2 \mathrm{~kJ} / \mathrm{mol}
\end{aligned}
$$

\section{Eigen ion}

Eigen ion is $\mathrm{H}_{9} \mathrm{O}_{4}^{+}=\mathrm{H}^{+} . . .4 \mathrm{H}_{2} \mathrm{O}$

2.1. The thermodynamic parameters for $\mathrm{H}_{9} \mathrm{O}_{4 \mathrm{gas}}^{+}$

Eigen ion is $\mathrm{H}_{5} \mathrm{O}_{2}^{+}=\mathrm{H}^{+} \ldots 2 \mathrm{H}_{2} \mathrm{O}$.

We write the formation of an Eigen ion in gas phase in the form of fourth reactions

$$
\begin{array}{cl} 
& \mathrm{H}^{+} . .+\mathrm{H}_{2} \mathrm{O} \rightarrow \mathrm{H}_{3} \mathrm{O}^{+} \\
& \mathrm{H}_{3} \mathrm{O}^{+}+\mathrm{H}_{2} \mathrm{O} \rightarrow \mathrm{H}_{5} \mathrm{O}_{2}^{+} \\
& \mathrm{H}_{5} \mathrm{O}_{2}^{+}+\mathrm{H}_{2} \mathrm{O} \rightarrow \mathrm{H}_{7} \mathrm{O}_{3}^{+} \\
& \mathrm{H}_{7} \mathrm{O}_{3}^{+}+\mathrm{H}_{2} \mathrm{O} \rightarrow \mathrm{H}_{9} \mathrm{O}_{4}^{+} \\
\hline & \mathrm{H}^{+}+4 \mathrm{H}_{2} \mathrm{O} \rightarrow \mathrm{H}_{9} \mathrm{O}_{4}^{+}
\end{array}
$$

Hence, $\Delta_{\mathrm{r}} H\left(\mathrm{H}^{+}+4 \mathrm{H}_{2} \mathrm{O} \rightarrow \mathrm{H}_{9} \mathrm{O}_{4}^{+}\right)_{\text {gas }}=-690.36-150.62-93.3-71.13=-1005.41 \mathrm{~kJ} / \mathrm{mol}$ and

$$
\Delta_{\mathrm{r}} G\left(\mathrm{H}^{+}+4 \mathrm{H}_{2} \mathrm{O} \rightarrow \mathrm{H}_{9} \mathrm{O}_{4}^{+}\right)_{\text {gas }}=-660-104.6-56.9-35.56=-857.06 \mathrm{~kJ} / \mathrm{mol}
$$

3.2. The thermodynamic functions of $\mathrm{H}_{9} \mathrm{O}_{4 \mathrm{gas}}^{+}$

For the reaction of formation of $\mathrm{H}_{9} \mathrm{O}_{4 \mathrm{gas}}^{+},\left(\mathrm{H}^{+}+4 \mathrm{H}_{2} \mathrm{O} \rightarrow \mathrm{H}_{9} \mathrm{O}_{4}^{+}\right)$, we write

$$
\begin{aligned}
\Delta_{\mathrm{r}} H^{\mathrm{o}} & =\Delta_{\mathrm{f}} H^{\mathrm{o}}\left(\mathrm{H}_{9} \mathrm{O}_{4 \mathrm{gas}}^{+}\right)-\Delta_{\mathrm{f}} H^{\mathrm{o}}\left(\mathrm{H}_{\text {gas }}^{+}\right)-4 \Delta_{\mathrm{f}} H^{\mathrm{o}}\left(\mathrm{H}_{2} \mathrm{O}_{\text {gas }}\right)= \\
& -1005.41=\Delta_{\mathrm{f}} H^{\mathrm{o}}\left(\mathrm{H}_{9} \mathrm{O}_{4 \text { gas }}^{+}\right)-1536.2+4 \cdot 241.8
\end{aligned}
$$

Hence

$$
\Delta_{\mathrm{f}} H^{\mathrm{o}}\left(\mathrm{H}_{9} \mathrm{O}_{4 \mathrm{gas}}^{+}\right)=-1005.41+1536.2-4241.8=-436.41 \mathrm{~kJ} / \mathrm{mol}
$$


Similarly, we find the values of the Gibbs function

$$
\begin{aligned}
\Delta_{\mathrm{r}} G^{\mathrm{o}}= & \Delta_{\mathrm{f}} G^{\mathrm{o}}\left(\mathrm{H}_{9} \mathrm{O}_{4 \mathrm{gas}}^{+}\right)-\Delta_{\mathrm{f}} G^{\mathrm{o}}\left(\mathrm{H}_{\mathrm{gas}}^{+}\right)-4 \cdot \Delta_{\mathrm{f}} G^{\mathrm{o}}\left(\mathrm{H}_{2} \mathrm{O}_{\mathrm{gas}}\right)= \\
& -857.06=\Delta_{\mathrm{f}} G^{\mathrm{o}}\left(\mathrm{H}_{9} \mathrm{O}_{4 \mathrm{gas}}^{+}\right)-1517+4 \cdot 228.6 \quad \text { and } \\
& \Delta_{\mathrm{f}} G^{\mathrm{o}}\left(\mathrm{H}_{9} \mathrm{O}_{4 \mathrm{gas}}^{+}\right)=-857.06+1517-4 \cdot 228.6=-254.46 \mathrm{~kJ} / \mathrm{mol}
\end{aligned}
$$

\subsection{Solvation}

We will consider the process of solvation of a proton in the form of two reactions: the addition of a proton to a fourth water molecules in the gas phase and the solvation of the formed ion $\mathrm{H}_{9} \mathrm{O}_{4 \mathrm{gas}}^{+}$. In the case of enthalpy we have

$$
\Delta_{\mathrm{S}} H^{\mathrm{o}}\left(\mathrm{H}^{+}\right)=\Delta_{\mathrm{r}} H^{\mathrm{o}}\left(\mathrm{H}^{+}+4 \mathrm{H}_{2} \mathrm{O}\right)_{\mathrm{gas}}+\Delta_{\mathrm{S}} H^{\mathrm{o}}\left(\mathrm{H}_{9} \mathrm{O}_{4 \mathrm{gas}}^{+}\right)=-1005.41+\Delta_{\mathrm{S}} H^{\mathrm{o}}\left(\mathrm{H}_{9} \mathrm{O}_{4 \mathrm{gas}}^{+}\right) .
$$

From the last equation we obtain

$$
\Delta_{\mathrm{S}} H^{\mathrm{o}}\left(\mathrm{H}_{9} \mathrm{O}_{4 \mathrm{gas}}^{+}\right)=\Delta_{\mathrm{S}} H^{\mathrm{o}}\left(\mathrm{H}^{+}\right)+1005.41=-1150.1+1005.41=-144.69 \mathrm{~kJ} / \mathrm{mol}
$$

Carrying out similar calculations for the Gibbs function,

$$
\begin{gathered}
\Delta_{\mathrm{S}} G^{\mathrm{o}}\left(\mathrm{H}^{+}\right)=-1104.5=\Delta_{\mathrm{r}} G^{\mathrm{o}}\left(\mathrm{H}^{+}+4 \mathrm{H}_{2} \mathrm{O} \rightarrow \mathrm{H}_{9} \mathrm{O}_{4 \mathrm{gas}}^{+}\right)_{\mathrm{gas}}+\Delta_{\mathrm{S}} G^{\mathrm{o}}\left(\mathrm{H}_{9} \mathrm{O}_{4 \mathrm{gas}}^{+}\right)= \\
\Delta_{\mathrm{S}} G^{\mathrm{o}}\left(\mathrm{H}_{9} \mathrm{O}_{4 \mathrm{gas}}^{+}\right)-857.06 \mathrm{~kJ} / \mathrm{mol} .
\end{gathered}
$$

we find

$$
\Delta_{\mathrm{S}} G^{\mathrm{o}}\left(\mathrm{H}_{9} \mathrm{O}_{4 \mathrm{gas}}^{+}\right)=-1104.5+857.06=-247.44 \mathrm{~kJ} / \mathrm{mol} \text {. }
$$

3.4. The thermodynamic parameters of $\mathrm{H}_{9} \mathrm{O}_{4 a q}^{+}$

Knowing the thermodynamic parameters of solvation and thermodynamic parameters in the gas phase we calculate the standard values of the thermodynamic parameters formation for $\mathrm{H}_{9} \mathrm{O}_{4 \mathrm{aq}}^{+}$in an aqueous solution.

$\Delta_{\mathrm{S}} H^{\mathrm{o}}\left(\mathrm{H}_{9} \mathrm{O}_{4}^{+}\right)=\Delta_{\mathrm{f}} H^{\mathrm{o}}\left(\mathrm{H}_{9} \mathrm{O}_{4 \mathrm{qq}}^{+}\right)-\Delta_{\mathrm{f}} H^{\mathrm{o}}\left(\mathrm{H}_{9} \mathrm{O}_{4 \mathrm{gas}}^{+}\right)$and

$\Delta_{\mathrm{f}} H^{\mathrm{o}}\left(\mathrm{H}_{9} \mathrm{O}_{4 \mathrm{aq}}^{+}\right)=\Delta_{\mathrm{S}} H^{\mathrm{o}}\left(\mathrm{H}_{9} \mathrm{O}_{4}^{+}\right)+\Delta_{\mathrm{f}} H^{\mathrm{o}}\left(\mathrm{H}_{9} \mathrm{O}_{4 \mathrm{gas}}^{+}\right)=-144.69-436.41=-581.1 \mathrm{~kJ} / \mathrm{mol}$

For $G$ function we have

$\Delta_{\mathrm{S}} G^{\mathrm{o}}\left(\mathrm{H}_{9} \mathrm{O}_{4}^{+}\right)=\Delta_{\mathrm{f}} G^{\mathrm{o}}\left(\mathrm{H}_{9} \mathrm{O}_{4 \mathrm{aq}}^{+}\right)-\Delta_{\mathrm{f}} G^{\mathrm{o}}\left(\mathrm{H}_{9} \mathrm{O}_{4 \mathrm{gas}}^{+}\right)$and

$\Delta_{\mathrm{f}} G^{\mathrm{o}}\left(\mathrm{H}_{9} \mathrm{O}_{4 \mathrm{aq}}^{+}\right)=\Delta_{\mathrm{S}} G^{\mathrm{o}}\left(\mathrm{H}_{9} \mathrm{O}_{4}^{+}\right)+\Delta_{\mathrm{f}} G^{\mathrm{o}}\left(\mathrm{H}_{9} \mathrm{O}_{4 \mathrm{gas}}^{+}\right)=-247.44-254.46=-501.9 \mathrm{~kJ} / \mathrm{mol}$ 
3.5. Transformed value

Transformed value is equal

$\Delta_{\mathrm{f}} G^{\mathrm{o}}\left(\mathrm{H}_{9} \mathrm{O}_{4 \mathrm{aq}}^{+}\right)=\Delta_{\mathrm{f}} G^{\mathrm{o}}\left(\mathrm{H}_{9} \mathrm{O}_{4 \mathrm{aq}}^{+}\right)+366.09[3]=-501.9+366.09=-135.81 \mathrm{~kJ} / \mathrm{mol}$

3.6. Convention values

Knowing the standard thermodynamic parameters of $\mathrm{H}_{9} \mathrm{O}_{4 a \mathrm{q}}^{+}$formation, we find similar values within the framework of the convention

$\Delta_{\mathrm{f}} H_{\mathrm{conv}}^{\mathrm{O}}\left(\mathrm{H}_{9} \mathrm{O}_{4 \mathrm{aq}}^{+}\right)=\Delta_{\mathrm{f}} H^{\mathrm{o}}\left(\mathrm{H}_{9} \mathrm{O}_{4 \mathrm{aq}}^{+}\right)-386.25=-581.1-386.25=-967.35 \mathrm{~kJ} / \mathrm{mol}$ and

$\Delta_{\mathrm{f}} G_{\mathrm{conv}}^{\mathrm{O}}\left(\mathrm{H}_{9} \mathrm{O}_{4 \mathrm{aq}}^{+}\right)=\Delta_{\mathrm{f}} G^{\mathrm{o}}\left(\mathrm{H}_{9} \mathrm{O}_{4 \mathrm{aq}}^{+}\right)-412.5=-501.9-412.5=-914.4 \mathrm{~kJ} / \mathrm{mol}$

\subsection{Cation $\mathrm{H}_{17} \mathrm{O}_{8}^{+}$}

4.1. The thermodynamic parameters for $\mathrm{H}_{17} \mathrm{O}_{8}^{+}$

Reaction $\quad \mathrm{H}^{+}+8 \mathrm{H}_{2} \mathrm{O} \rightarrow \mathrm{H}_{17} \mathrm{O}_{8}^{+}$

Hence due to [4], $\Delta_{\mathrm{r}} H\left(\mathrm{H}^{+}+8 \mathrm{H}_{2} \mathrm{O} \rightarrow \mathrm{H}_{17} \mathrm{O}_{8}^{+}\right)=-1215.87 \mathrm{~kJ} / \mathrm{mol}$ and

$$
\Delta_{\mathrm{r}} G=-917.32 \mathrm{~kJ} / \mathrm{mol}
$$

4.2. The thermodynamic functions of $\mathrm{H}_{17} \mathrm{O}_{8}^{+}$

For the reaction of formation of $\mathrm{H}_{17} \mathrm{O}_{8 \text { gas }}^{+}$, we write

$$
\begin{aligned}
\Delta_{\mathrm{r}} H^{\mathrm{o}} & =\Delta_{\mathrm{f}} H^{\mathrm{o}}\left(\mathrm{H}_{17} \mathrm{O}_{8}^{+}\right)-\Delta_{\mathrm{f}} H^{\mathrm{o}}\left(\mathrm{H}_{\text {gas }}^{+}\right)-8 \Delta_{\mathrm{f}} H^{\mathrm{o}}\left(\mathrm{H}_{2} \mathrm{O}_{\text {gas }}\right)= \\
& -1215.87=\Delta_{\mathrm{f}} H^{\mathrm{o}}\left(\mathrm{H}_{17} \mathrm{O}_{8 \mathrm{gas}}^{+}\right)-1536.2+8 \cdot 241.8
\end{aligned}
$$

Hence

$$
\Delta_{\mathrm{f}} H^{\mathrm{o}}\left(\mathrm{H}_{17} \mathrm{O}_{8 \mathrm{gas}}^{+}\right)=-1215.87+1536.2-8241.8=-1614.07 \mathrm{~kJ} / \mathrm{mol}
$$

Similarly, we find the values of the Gibbs function

$$
\begin{aligned}
\Delta_{\mathrm{r}} G^{\mathrm{o}}= & \Delta_{\mathrm{f}} G^{\mathrm{o}}\left(\mathrm{H}_{17} \mathrm{O}_{8}^{+}\right)-\Delta_{\mathrm{f}} G^{\mathrm{o}}\left(\mathrm{H}_{\text {gas }}^{+}\right)-4 \cdot \Delta_{\mathrm{f}} G^{\mathrm{o}}\left(\mathrm{H}_{2} \mathrm{O}_{\text {gas }}\right)= \\
& -917.32=\Delta_{\mathrm{f}} G^{\mathrm{o}}\left(\mathrm{H}_{17} \mathrm{O}_{8 \mathrm{gas}}^{+}\right)-1517+8 \cdot 228.6 \quad \text { and } \\
& \Delta_{\mathrm{f}} G^{\mathrm{o}}\left(\mathrm{H}_{17} \mathrm{O}_{8 \mathrm{gas}}^{+}\right)=-917.32+1517-8 \cdot 228.6=-1229.12 \mathrm{~kJ} / \mathrm{mol}
\end{aligned}
$$




\subsection{Solvation}

We will consider the process of solvation of a proton in the form of two reactions: the addition of a proton to a 8 water molecules in the gas phase and the solvation of the formed ion $\mathrm{H}_{17} \mathrm{O}_{8 \mathrm{gas}}^{+}$.

In the case of enthalpy we have

$$
\Delta_{\mathrm{S}} H^{\mathrm{o}}\left(\mathrm{H}^{+}\right)=\Delta_{\mathrm{r}} H^{\mathrm{o}}\left(\mathrm{H}^{+}+8 \mathrm{H}_{2} \mathrm{O}\right)_{\mathrm{gas}}+\Delta_{\mathrm{S}} H^{\mathrm{o}}\left(\mathrm{H}_{17} \mathrm{O}_{8 \mathrm{gas}}^{+}\right)=-1215.87+\Delta_{\mathrm{S}} H^{\mathrm{o}}\left(\mathrm{H}_{17} \mathrm{O}_{8 \mathrm{gas}}^{+}\right) .
$$

From the last equation we obtain

$$
\Delta_{\mathrm{S}} H^{\mathrm{o}}\left(\mathrm{H}_{17} \mathrm{O}_{8 \mathrm{gas}}^{+}\right)=\Delta_{\mathrm{S}} H^{\mathrm{o}}\left(\mathrm{H}^{+}\right)+1215.87=-1150.1+1215.87=+65.77 \mathrm{~kJ} / \mathrm{mol}
$$

\section{Summation:}

\begin{tabular}{|c|c|c|c|c|c|c|c|c|c|c|}
\hline $\begin{array}{l}\mathrm{N} \\
\mathrm{o}\end{array}$ & Ion & $\begin{array}{c}\Delta_{\mathrm{f}} H_{\text {gas }}^{\mathrm{o}} \\
\mathrm{kJ} / \mathrm{mol}\end{array}$ & $\begin{array}{c}\Delta_{\mathrm{f}} G_{\mathrm{gas}}^{\mathrm{o}} \\
\mathrm{kJ} / \mathrm{mol}\end{array}$ & $\begin{array}{c}\Delta_{S} H^{\mathrm{o}} \\
\mathrm{kJ} / \mathrm{mol}\end{array}$ & $\begin{array}{c}\Delta_{S} G^{\mathrm{o}} \\
\mathrm{kJ} / \mathrm{mol}\end{array}$ & $\Delta_{\mathrm{f}} H_{\mathrm{aq}}^{\mathrm{o}}$ & $\begin{array}{c}\Delta_{\mathrm{f}} G_{\mathrm{aq}}^{\mathrm{o}} \\
\mathrm{kJ} / \mathrm{mol}\end{array}$ & $\begin{array}{c}\Delta_{\mathrm{f}} G_{\text {aq }}^{\mathrm{J}} \\
\mathrm{kJ} / \mathrm{mol}\end{array}$ & $\begin{array}{c}\Delta_{\mathrm{f}} H_{\text {aq }}^{\mathrm{o}} \\
\mathrm{conv} \\
\mathrm{kJ} / \mathrm{mol}\end{array}$ & $\begin{array}{c}\Delta_{\mathrm{f}} G_{\text {aq }}^{\mathrm{o}} \\
\mathrm{conv} \\
\mathrm{kJ} / \mathrm{mol}\end{array}$ \\
\hline 1 & $\mathrm{H}^{+}$ & 1536.2 & 1517 & -1150.1 & -1104.5 & 386.25 & 412.5 & 452.46 & 0 & 0 \\
\hline 2 & $\mathrm{H}_{3} \mathrm{O}^{+}$ & 604.04 & 628.4 & -459.74 & -444.5 & 144.3 & 183.9 & 305.39 & -241.95 & -228.6 \\
\hline 3 & $\mathrm{H}_{5} \mathrm{O}_{2}^{+}$ & 211.62 & 295.2 & -309.12 & -333.9 & -97.5 & -38.7 & 164.33 & -483.75 & -451.2 \\
\hline 4 & $\mathrm{H}_{9} \mathrm{O}_{4}^{+}$ & -436.41 & -254.46 & -144.69 & -247.44 & -581.1 & -501.9 & -135.81 & -967.35 & -914.4 \\
\hline 5 & $\mathrm{H}_{17} \mathrm{O}_{8}^{+}$ & -1614.07 & -1229.12 & +65.77 & & & & & & \\
\hline
\end{tabular}

The parameters for $\mathrm{H}^{+}$are taken from [2].

Thermodynamic functions in the convention version were calculated in accordance with [2]

$$
\Delta_{\mathrm{f}} H_{\mathrm{aq}}^{\mathrm{o}} \text { conv }=\Delta_{\mathrm{f}} H_{\mathrm{aq}}^{\mathrm{o}}-386.25 \mathrm{~kJ} / \mathrm{mol} \quad \Delta_{\mathrm{f}} G_{\mathrm{aq}}^{\mathrm{o}} \text { conv }=\Delta_{\mathrm{f}} G_{\mathrm{aq}}^{\mathrm{o}}-412.5 \mathrm{~kJ} / \mathrm{mol}
$$

\section{References}

1. Handbook of Chemistry and Physics, Haynes W. M., Editor-in-Chief, Lido D.R., Bruno T.J. associate Editors, 95th ed.; CRC Press, New York, 2014-2015.

2. Bazhin N. M. Standard Values of the Thermodynamic Functions of the Formation of Ions in an Aqueous Solution and their Change during Solvation. J. Phys. Chem. A2020, 124, 11051-11060.

3. Alberty R. A., Thermodynamics of Biochemical Reactions, Wiley, USA, 2003.

4. P. Kebarle, S. K. Searles, A. Zolla, J. Scarborough, and M. Arshadi The Solvation of the Hydrogen Ion by Water Molecules in the Gas Phase. Heats and Entropies of Solvation of Individual Reactions: $\mathrm{H}+(\mathrm{H} 20)_{\mathrm{n}-1}$, JACS vol 89. No 25 (1967) 6393 - 6399 